\title{
Treatment of Influenza Virus Infections with Chinese Medicine
}

\author{
Da Yong $\mathrm{Lu}^{1 *}$, Ting Ren $\mathrm{Lu}^{2}$, Hong Ying $\mathbf{W u}^{2}$ \\ ${ }^{1}$ School of Life Sciences, Shanghai University, Shanghai 200444, PR China \\ ${ }^{2}$ College of Science, Shanghai University, Shanghai 200444, PR China
}

As the concern about the deadliest pandemic viruses, such as bird flu, is being intensified [1-3], prompt and effective treatments of viral infected patients are in high demand. Influenza virus has been catastrophic in the history and currently large-scale disease outbreaks in southeast China, Asia, and Netherlands has attracted the attention of virology researchers and sociologists. In the two recently largest history records of 1968 and 1918, 750,000 and 40 million people respectively died of the catastrophic outbreak of some deadliest influenza virus strains [4-5]. The pandemics of the past century includes the catastrophic H1N1 Spanish influenza of 1918, and H2N2 Asian flu of 1957 (more than million deaths globally), and the H3N2 Hong Kong flu of 1968 (-0.5 million deaths globally) [6-7]. Avian flu virus is one of these influenza viruses, and human's fear for avian flu is therefore no less than those of other deadliest viruses, such as HIV/AIDS and SARS (severe acute respiratory syndrome). Since the re-outbreak of avian flu endemic might take place at any geographical location on Earth at any time in future and the completed effective therapeutic means have not been available worldwide, therefore it is no wonder why there are growing worries among world-leading virologists, epidemiologists and political leaders who are endeavoring to handle avian flu spread seriously.

\section{Current Treatment Means All Over the World}

Currently, the most usable and effective way to the prevention of the avian influenza endemic is to remove the virus spreading sources culling and slaughtering the birds, chickens and ducks on large scales [2] and isolate and treat patients with vaccines, neuraminidase inhibitors and/or antivirals individually at the same time [8-10].

Antiviral drugs ought to be a major countermeasure to reduce the spread of virus in humans. Now common types of the antiviral drugs are as follows:

1. Virus uptake inhibitors (M2 ion channel blockers); Adamantanes, Amantadine and Rimantadine

2. Transcription replication inhibitors; Ribavirin, Viramidine, T-705, antisense DNA oligomers and interfering RNA

3. Sialic acidGbinding inhibitors (neuraminidase inhibitors); Oseltamivir, Zanamivir, Peramivir; they are more useful in early stage of disease, and resistance to this type of drug is relatively less common, but has been reported $[6,9]$.

\section{Major Problems of Current Therapeutic Means}

Major problems of current therapeutic means are only effective to early stage of disease of these therapies and there is increasing reports of drug resistance [11]. There is also high mortality rate in some highly-infectious and highlygtloxic virus strain outbreak and sometimes the mortality rates or infected patients can be higher than 30\% [12]. Since the avian virus is sometimes highly infectious, patients who suffer may be large volume in population; one percent increase of mortality rates may cost hundreds or even thousands of lives. Thus highly effective therapies are in high demands. One of them is by using traditional Chinese medicine.

\section{Treatment of Virus Infection by Traditional Chinese Medicine}

China has a long history of using of traditional Chinese medicine for the treatment of the spread of large-scale cold (similar like avian flu) endemics, which probably makes China the most populated country in the world. It has been effectively used since ZhongGDin Zhang (AD150219) in China [12]. Traditional Chinese medicine can combat the disease by strengthening, modulating and offsetting damaged organs and "Yang and Ying" with a formulated herb. There are several formulated soups for cold (infected) fever (similarity to avian flu symptoms) according to Zhang, including "GuiG[Zhi Soup", "Ma-Huang Soup", "DaQingG[long Soup" and "GeGGeng Soup" [13]. It is a cheaper way and herbs are available all the times. This theory has been successively controlling a lot of virus-infected endemics throughout in the long history of China. We propose that this type of treatment should also be further studied and used. The most frequently used prescription in the group of expelling exogenous wind $\exists$ Theat was Yin $\exists[$ Qiao $\exists$ \$ $\$$ an. And the prescription of expelling exogenous wind-cold was Jing-Fang-Bai-Du-San. The

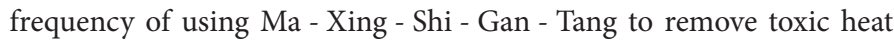
obstruction in the lungs was up to $100 \%$ [14-17]. This treatment options can also be used as the preventive therapy of large human population in potential avian flu epidemic conditions.

\section{Importance of Traditional Chinese Medicine}

Treatment of virus infection by Chinese medicine has two thousand years history. It has three advantageous (i) it targets the disease severe symptoms. It is the disease severe symptoms that lead to patients' death; (ii) the recipe of traditional Chinese medicine is of multiple components. Thus it is not easy to produce drug resistance and have combined effects in virus-treatment. (iii) High reputation of traditional Chinese medicine. There are a lot of successful examples in prevent large scale virus-disease endemic in Chinese history. It means that traditional Chinese medicine may be used in future epidemics.

\section{Future Direction}

However, traditional Chinese medicine is not familiar in Western world and it can not be practiced in those countries. We believe these conditions can be improved by further studies and communications between the two cultures. There are a lot of studies to do if we think it is useful and deserved.

*Corresponding author: Da Yong Lu, Associate professor, School of Life Sciences, Shanghai University, China, E-mail: ludayong@sh163.net

Received May 01, 2012; Accepted May 03, 2012; Published May 07, 2012

Citation: Yong Lu D, Ren Lu T,Ying Wu H (2012) Treatment of Influenza Virus Infections with Chinese Medicine. Adv Pharmacoepidem Drug Safety 1:e104. doi:10.4172/2167-1052.1000e104

Copyright: ( $) 2012$ Yong Lu D, et al. This is an open-access article distributed under the terms of the Creative Commons Attribution License, which permits unrestricted use, distribution, and reproduction in any medium, provided the original author and source are credited. 
Citation: Yong Lu D, Ren Lu T,Ying Wu H (2012) Treatment of Influenza Virus Infections with Chinese Medicine. Adv Pharmacoepidem Drug Safety 1:e104. doi:10.4172/2167-1052.1000e104

Page 2 of 2

\section{References}

1. Abbott A, Pearson H (2004) Fear of human pandemic grows as bird flu sweeps through Asia. Nature 427: 472- 473.

2. Webster R, Hulse D (2005) Controlling avian flu at the source. Nature 435: 415-418.

3. Fouchier R, Kuiken T, Rimmelzwaan G, Osterhaus A (2005) Global task force for influenza. Nature 435: 419-420.

4. Aldhous P, Tomlin S (2005) Avian flu special: avian flu: are we ready? Nature 435: 399.

5. Osterholm MT (2005) Preparing for the next pandemic. N Engl J Med 352: 1839-1842.

6. Salomon R, Webster RG (2009) The influenza virus enigma. Cell 136: 402-410.

7. Lewis DB (2006) Avian flu to human influenza. Annu Rev Med 57: 139-154.

8. Fauci AS (2005) Race against time. Nature 435: 423-424.

9. Beigel J, Bray M (2008) Current and future antiviral therapy of severe seasonal and avian influenza. Antiviral Res 78: 91-102.

10. Smith JR (2010) Oseltamivir in human avian influenza infection. J Antimicrob Chemther 65: ii25-ii33.
11. Orozovic G, Orozovic K, Lennerstrand J, Olsen B (2011) Detection of resistance mutations to antivirals Oseltamivir and Zanamivir in avian influenza A viruses isolated from wild birds. PLoS One. 6: e16028.

12. Lu DY, Lu TR, Wu HY (2012) Avian flu, pathogenesis and therapy. Anti-Infective Agents 10: 124-129.

13. Zhang, Z.J. (2005) Cold Factors and Treatments People's Medical and Public Health Publication.

14. Liu DF, Liu YL, Chen $H$, Zeng WL, Wen XM, et al. (2011) Evaluation on the curative effect and safety of Chinese traditional medicine in treatment of mild a/hH1N1 influenza in sichuan area. Modern Preventive Medicine 38: 338-343.

15. Zhou Ho, Tao LT, Xu HC, Jiang YG, Deng YQ, et al. (2011) Discussion on Laws of Traditional Chinese Medical Treatment of H1N1 Influenza Based on Cohort Study. World Science and Technology/Modernization of Traditional Chinese Medicine and Materia Medica 13: 777-782.

16. Rumschlag-Booms E, Zhang HJ, Soejarto DD, Fong HHS, Rong LJ (2011) Development of an antiviral screening protocol: one-stone-two-birds. J Antivir Antiretrovir 7: 8-10.

17. Li YH, Yan QB, Yu KZ, Tong HM, Sun Y (2008) Study of the compound Chinese medicine against the anti-avian influenza virus. Scientia Agricultura Sinica 41: 1511-1518. 